\title{
Quantifying FDG uptake to diagnose cardiac device infections: When and how should we do it?
}

\author{
Ron Blankstein, MD, FACC, FASNC, ${ }^{\mathrm{a}, \mathrm{b}}$ and Edward J. Miller, MD, PhD, FASNC \\ a Non-Invasive Cardiovascular Imaging Program, Departments of Medicine (Cardiovascular \\ Division) and Radiology, Brigham and Women's Hospital, Boston, MA \\ ${ }^{\mathrm{b}}$ Harvard Medical School, Boston, MA \\ c Section of Cardiovascular Medicine, Departments of Medicine and Radiology, Yale University \\ School of Medicine, New Haven, CT
}

Received Sep 15, 2015; accepted Sep 15, 2015

doi: 10.1007/s12350-015-0293-2

\section{See related article, pp.1457-1466}

The use of cardiac implantable electronic devices (CIEDs) has increased worldwide since the 1960s and now includes implantable cardiac defibrillators, cardiac resynchronization therapy devices, and permanent pacemakers. While such devices can offer significant benefit to patients, recent data have suggested that the incidence of CIED infections is increasing. Exacerbating this problem is the fact that CIED infections can be difficult to diagnose, as traditional imaging techniques such as echocardiography have poor sensitivity and blood cultures may be negative. Once the diagnosis is established, device extraction is often required, which is a costly procedure that is associated with significant risks.

Recently, positron emission tomography (PET) using F18-Fluorodeoxyglucose (FDG) has been used to image various cardiovascular infections, including CIED $^{1-3}$ as well as prosthetic valves. ${ }^{4}$ Consequently, the recent 2015 European Society of Cardiology (ESC) Guidelines for the management of infective endocarditis (IE) have added FDG PET imaging, with the argument that FDG PET can improve the sensitivity of the modified Duke criteria when there is possible IE or rejected IE with a high suspicion. ${ }^{5}$ The ESC guidelines propose

Reprint requests: Ron Blankstein, MD, FACC, FASNC, Non-Invasive Cardiovascular Imaging Program, Departments of Medicine (Cardiovascular Division) and Radiology, Brigham and Women's Hospital, 75 Francis St, Boston, MA 02115; rblankstein@partners.org J Nucl Cardiol 2016;23:1467-9.

$1071-3581 / \$ 34.00$

Copyright $\varnothing 2015$ American Society of Nuclear Cardiology. that in the setting of suspected endocarditis on a prosthetic valve, abnormal FDG activity or radiolabeled leucocyte SPECT/CT should be considered a major criterion, if the prosthesis was implanted $>3$ months previously. Supporting these recommendations, a recent study by Pizzi et $\mathrm{al}^{6}$ evaluated 92 patients with suspected prosthetic valve or cardiac device IE and found that combining the modified Duke Criteria with FDG PET findings increased the sensitivity to detect IE from $52 \%$ to $91 \%$ and resulted in a conclusive diagnosis for $95 \%$ of the patients.

However, protocols for the acquisition as well as interpretation of FDG PET studies to evaluate infections have not been standardized. For instance, there is variability in incubation time post FDG injection (e.g., 60120 minutes). In addition, the interpretation of FDG PET studies is often performed using a qualitative visual read. Developing standardized acquisition and quantitative interpretive approaches may offer several advantages, such as improved accuracy for differentiating a true infection from non-specific uptake. While quantitative assessment of FDG uptake may help assess the response to therapy in some cases of IE, it is noteworthy that complete device removal is indicated for all cases of CIED infections. Future studies may also determine if quantitative data from FDG PET studies, combined with imaging data from echocardiography or cardiac CT, may help predict prognosis or the risk of complications such as embolism.

Realizing the potential advantages of developing quantitative approaches to interpret PET FDG scans, in this issue of the Journal of Nuclear Cardiology, Memmott et $\mathrm{al}^{7}$ compared the accuracy of various quantitative approaches in relation to incubation duration used to evaluate patients with suspected cardiac implantable electronic devices infections. They analyzed 
80 FDG PET scans from 78 patients, of whom 41 were asymptomatic patients referred for oncological reason. Among the remaining 37 patients who had suspected cardiac device infections, 13 were deemed not to have an infection upon follow-up, while 24 were confirmed to have an infection.

In addition to visual analysis, various quantitative measurements were performed (see Table 1 for details), including both the SUV maximum as well as various semi-quantitative ratios which normalize the maximal count to background activity.

All measures of SUVmax (regardless of whether or how correction for background activity was performed) showed a high accuracy for discriminating patients with infected devices from those who were asymptomatic and were evaluated for oncologic reasons. However, when patients with infected devices were compared to those who had an initial suspicion for infection but then were determined to be infection free upon follow-up (i.e., the comparison which is clinically most relevant), the accuracy was lower, although the AUC remained above 0.90 for most techniques. While the authors suggested that the highest AUC values were found when using the SUV max corrected for hepatic blood pool (HBP) activity, measured at 180 minutes, the relatively small numbers of patients in these groups do not allow for any definitive conclusions (for example, the AUC for SUVmax at 90 minutes was 0.960 .03 , while for SUVmax/SUVmax of HBP the AUC was 0.97 0.02).

Even though the current results do not support a definitive need for correcting the SUVmax by background activity, this study supports the concept that a quantitative assessment of SUVmaximum can be useful for discriminating between patients who have device infections from those who do not. These quantitative techniques may be especially relevant when assessing response to therapy for an individual patient. While this could be performed in some patients who have infected CIED, the majority of patients with CIED infections require device extraction rather than serial follow-up. This is in contrast to other conditions, such as treatment of malignancies, treatment of vasculitis, or treatment of cardiac sarcoidosis, ${ }^{8,9}$ where the need to follow response to therapy is more relevant. In such scenarios, it is essential to use the same acquisition and image analysis techniques. ${ }^{10}$

With respect to identifying patients with infected devices, it should be recognized that there is no single

Table 1. Methods used to determine F18-fluorodeoxyglucose uptake in patients with suspected cardiac implantable electronic device infections

$\begin{array}{lll}\text { Technique } & \text { ROI Placement Comment }\end{array}$

1. Visual read

2. SUV Maximum alone (SUVmax)

\section{SUVmax/SUVmax in contralateral side}

\section{SUVmax/SUVmax in lung parenchyma}

5. SUVmax/SUVmax in hepatic blood pool (HBP)

6. SUVmax/SUVmax in mediastinal blood pool (MBP)
N/A

Quick

Relies on experience

May be limited for comparing serial studies

Measure the highest uptake of FDG, either around the device pocket or along the lead(s)

ROI placed over soft tissue in an area which mirrored the location of the device

ROI places over area of uniform parenchyma for each lung

ROI placed over homogenous area within the right lobe of the liver

ROI placed in aortic arch
Reproducible and quick Use non-attenuation-corrected images to avoid artifacts from attenuation correction of dense objects

Avoid peripheral areas of high intensity in the skin

Average of both lungs used

Avoid any areas of calcifications of the vessel wall 
SUVmax threshold that can be used to discriminate patients who have CIED infections from those who do not. Indeed, the authors show that SUV thresholds increase with the time of imaging post FDG injection (e.g., incubation time; from 60 to 180 minutes). Centers should have thresholds that are dependent on their protocols, as specifics such as incubation time, image acquisition details (e.g., use of metal artifact reduction software), the quality of the CT used for attenuation correction, and image analysis methodology (e.g., placement of ROI) can all have a significant impact on SUV measurements.

While the use of FDG imaging provides a useful technique for assessing patients with suspected device infections, there are caveats to be aware of: (a) falsepositive findings can occur from post-surgical inflammation, thrombi, tumors, or metabolically active plaques; (b) integration of FDG PET data together with other imaging and clinical data is often required; and (c) careful attention is needed in developing and following institutional imaging protocols.

While the current study does not assess if quantitative techniques such as measuring the SUVmax improve the accuracy afforded by a visual assessment, given the study results, SUVmax should be routinely performed and reported when assessing for cardiac device infections. While the absence of any FDG uptake will be associated with a high negative predictive value, a high SUVmax (i.e., >3-4) is highly specific for infections. Nevertheless, in some patients borderline values may be obtained and, in such cases, deciding whether an infection is present or absent may remain unknown even after FDG PET imaging. While some reports have suggested that delayed imaging could be useful by increasing metabolic uptake of FDG, the current study shows that compared to imaging at 90 minutes, imaging performed at 180 minutes post FDG injection results in increased SUVmax for both patients with and without infections, and thus the rate of increase cannot be used to improve the identification of patients with CIED infections.

In conclusion, while the study by Memmott et $\mathrm{al}^{7}$ provides useful data on the use of FDG PET for evaluating patients with suspected CIED infections, further research is needed for developing and validating accurate and reproducible techniques, which, when combined with clinical data, will improve the diagnosis of CIED infections as well as provide clinically relevant prognostic data.

\section{Conflicts of interests}

There are no disclosures or conflicts of interests.

\section{References}

1. Graziosi M, Nanni C, Lorenzini M, Diemberger I, Bonfiglioli R, Pasquale F, et al. Role of (1)(8)F-FDG PET/CT in the diagnosis of infective endocarditis in patients with an implanted cardiac device: A prospective study. Eur J Nucl Med Mol Imaging 2014;59:161723 .

2. Sarrazin JF, Philippon F, Tessier M, Guimond J, Molin F, Champagne $\mathbf{J}$, et al. Usefulness of fluorine-18 positron emission tomography/computed tomography for identification of cardiovascular implantable electronic device infections. J Am Coll Cardiol 2012;59:1616-25.

3. Ploux S, Riviere A, Amraoui S, Whinnett Z, Barandon L, Lafitte S, et al. Positron emission tomography in patients with suspected pacing system infections may play a critical role in difficult cases. Heart Rhythm 2011;8:1478-81.

4. Saby L, Laas O, Habib G, Cammilleri S, Mancini J, Tessonnier L, et al. Positron emission tomography/computed tomography for diagnosis of prosthetic valve endocarditis: Increased valvular 18 F-fluorodeoxyglucose uptake as a novel major criterion. J Am Coll Cardiol 2013;61:2374-82.

5. Habib G, Lancellotti P, Antunes MJ, Bongiorni MG, Casalta J-P, Del Zotti $F$ et al. 2015 ESC guidelines for the management of infective endocarditis: The task force for the management of infective endocarditis of the European Society of Cardiology (ESC)Endorsed by: European Association for Cardio-Thoracic Surgery (EACTS), the European Association of Nuclear Medicine (EANM). Eur Heart J 2015.

6. Pizzi MN, Roque A, Fernandez-Hidalgo N, Cuellar-Calabria H, Ferreira-Gonzalez I, Gonzalez-Alujas MT, et al. Improving the diagnosis of infective endocarditis in prosthetic valves and intracardiac devices with 18F-FDG-PET/CT-angiography: Initial results at an infective endocarditis referral center. Circulation 2015;132:1113-26.

7. Memmott MJ, James J, Armstrong IS, Tout D, Ahmed F. The performance of quantitation methods in the evaluation of cardiac implantable electronic device (CIED) infection: A technical review. J Nucl Cardiol 2015. doi:10.1007/s12350-015-0106-7.

8. Osborne MT, Hulten EA, Singh A, Waller AH, Bittencourt MS, Stewart GC, et al. Reduction in 18F-fluorodeoxyglucose uptake on serial cardiac positron emission tomography is associated with improved left ventricular ejection fraction in patients with cardiac sarcoidosis. J Nucl Cardiol 2014;21:166-74.

9. Ahmadian A, Brogan A, Berman J, Sverdlov AL, Mercier G, Mazzini M, et al. Quantitative interpretation of FDG PET/CT with myocardial perfusion imaging increases diagnostic information in the evaluation of cardiac sarcoidosis. J Nucl Cardiol 2014;21:92539.

10. Waller AH, Blankstein R. Quantifying myocardial inflammation using F18-fluorodeoxyglucose positron emission tomography in cardiac sarcoidosis. J Nucl Cardiol 2014;21:940-3. 